\title{
关于丙酮碘化反应实验的方法误差的讨论
}

韩莉 ${ }^{*}$, 金金金, 张卫

上海交通大学化学化工学院, 上海 200240

摘要: 用简单的方法估算了丙酮碘化反应实验中用平均速率代替瞬时速率产生的方法误差, 相对误差的绝对值为 $10^{-4}$ 数 量级, 并分析了产生误差的原因。

关键词：丙酮硔化反应；平均速率；瞬时速率；相对误差

中图分类号: G64; O6

\section{Discussion on Methodic Error of the Reaction between Acetone and lodine}

Li Han *, Xin Jin, Wei Zhang

School of Chemistry and Chemical Engineering, Shanghai Jiao Tong University, Shanghai 200240, P. R. China.

Abstract: The methodic error of reaction between acetone and iodine was investigated using a simple and novel method. The relative error was calculated to as low as $10^{-4}$. We also give a discussion on the reason causing this error.

Key Words: Reaction between acetone and iodine; Mean reaction rate; Momentary reaction rate; Relative error

研究化学反应的速率方程式是普通化学和无机化学实验课程重要的教学内容之一，最常使用的 是丙酮的碘化反应和过二硫酸铵与碘化钾反应这两种体系。某实验条件下的瞬时速率需要连续测定 很多数据作曲线才能得到, 测定反应的平均速率则比较简单, 面对一年级学生的动力学实验重点在 于更好地理解速率方程式, 节约实验时间并突出实验重点, 所以上海交通大学、吉林大学、浙江大 学、复旦大学、北京师范大学等国内许多高校的实验教材中都用平均速率代替瞬时速率 ${ }^{[1-5]}$ 。

学生们处理数据后分析误差原因时, 主要观点集中在 “平均速率代替瞬时速率这个方法本身带 来的误差”。平均速率代替瞬时速率产生的误差到底有多大? 虽然受限于大一学生的高等数学基础, 不能定量计算出误差大小, 但也应该对实验体系误差估算一下才能下结论。本文用比较简单的方法 讨论和估算了丙酮碘化反应中这个方法误差的大小。

在酸性水溶液中, 丙酮与碘发生如式(1)表示的丙酮碘化反应:

$$
\mathrm{CH}_{3} \mathrm{COCH}_{3}(\mathrm{aq})+\mathrm{I}_{2}(\mathrm{aq}) \rightarrow \mathrm{CH}_{3} \mathrm{COCH}_{2} \mathrm{I}(\mathrm{aq})+\mathrm{H}^{+}(\mathrm{aq})+\mathrm{I}^{-}(\mathrm{aq})
$$

丙酮碘化反应是一个复合反应, 在丙酮碘化反应实验的条件下反应速率是丙酮的一级反应, 与 碘的浓度无关，即速率方程为 $v=k c_{\text {(acetone) }}{ }^{[6]}$, 图 1 是一级反应反应物浓度与反应时间关系示意图。 反应开始时 (0 时刻)的瞬时速率 $v_{0}$ 等于曲线在 0 时刻(A 点)的切线的斜率的绝对值，反应在 $t$ 时刻的 瞬时速率 $v_{t}$ 等于曲线在 $t$ 时刻(C 点)的切线的斜率的绝对值, $0-t$ 时间段的平均速率 $\bar{v}$ 等于直角三角

收稿: 2019-05-16; 录用: 2019-06-27; 网络发表: 2019-07-08

“通讯作者, Email: lihan@sjtu.edu.cn

基金资助：上海交通大学教学发展中心教学发展基金(CTLD18B 0003) 
形 $\mathrm{ABC}$ 斜边 $\mathrm{AC}$ 的斜率的绝对值。由图 1 可以看出, 平均速率 $\bar{v}$ 大小一定在 $v_{0}$ 与 $v_{t}$ 之间, 所以平均 速率 $\bar{v}$ 与 0 时刻瞬时速率 $v_{0}$ 之间的误差的绝对值一定小于或等于 (时间 $t$ 无限短时等于) $t$ 时刻的瞬时 速率 $v_{t}$ 与 0 时刻瞬时速率 $v_{0}$ 之间误差的绝对值, 大小关系如式(2)表示:

$$
\left|\bar{v}-v_{0}\right| \leq\left|v_{t}-v_{0}\right|
$$

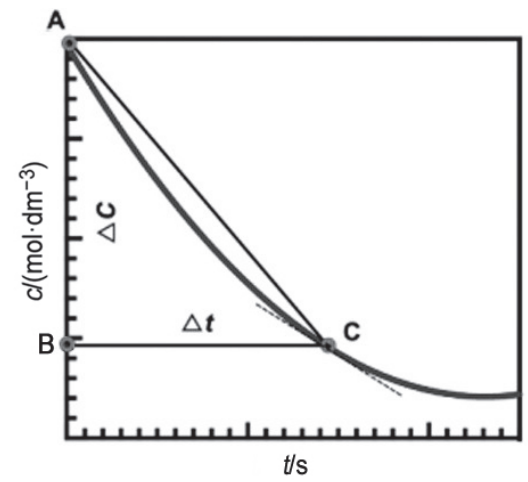

图 1 一级反应反应物浓度与反应时间关系示意图

某一组反应物浓度配比时的实验步骤 ${ }^{77}$ 如下: 在一个碘量瓶中加入 $4.00 \mathrm{~mol} \cdot \mathrm{dm}^{-3}$ 丙酮溶液 $10.0 \mathrm{~cm}^{3}$ 、 $1.00 \mathrm{~mol} \cdot \mathrm{dm}^{-3}$ 盐酸溶液 $10.0 \mathrm{~cm}^{3}$ 和去离子水 $15.0 \mathrm{~cm}^{3}$, 放入摚拌子。在搅拌下倒入 $0.00118 \mathrm{~mol} \cdot \mathrm{dm}^{-3}$ 碘溶液 $15.0 \mathrm{~cm}^{3}$, 同时开始计时。至反应体系溶液变为无色时停止计时, 记录消耗的时间 $t$ 。

下面我们来研究和估算一下上述体系中用 $0-t$ 时间段平均速率 $\bar{v}$ 代替 0 时刻瞬时速率 $v_{0}$ 带来的 误差有多大。根据该反应的速率方程式:

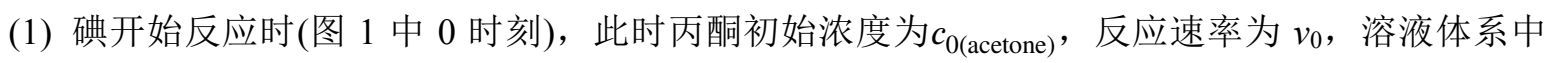
丙酮初始量为:

则:

$$
4.00 \times 10.0 \times 10^{-3}=4.00 \times 10^{-2}(\mathrm{~mol})
$$

$$
v_{0}=k c_{0(\text { acetone })}=k\left(\frac{4.00 \times 10^{-2}}{50.0 \times 10^{-3}}\right)=0.800 k\left(\mathrm{~mol} \cdot \mathrm{dm}^{-3} \cdot \mathrm{s}^{-1}\right)
$$

(2) 碘反应完(图 1 中 $t$ 时刻), 此时丙酮浓度为 $c_{t \text { (acetone) }}$, 反应速率为 $v_{t}$, 根据式(1)中的计量关 系, 碘全部反应完成消耗的丙酮量为:

则:

$$
0.00118 \times 15.0 \times 10^{-3}=1.77 \times 10^{-5}(\mathrm{~mol})
$$

$$
v_{t}=k c_{t(\text { acetone })}=k\left(\frac{4.00 \times 10^{-2}-1.77 \times 10^{-5}}{50.0 \times 10^{-3}}\right)=0.7996 k\left(\mathrm{~mol} \cdot \mathrm{dm}^{-3} \cdot \mathrm{s}^{-1}\right)
$$

(3) 误差大小估算。

$v_{t}$ 与 $v_{0}$ 之间的相对误差为:

$$
\frac{k c_{t(\text { acetone) }}-k c_{0 \text { (acetone) }}}{k c_{0(\text { acetone })}} \times 100 \%=-0.0443 \%
$$

由式(2)可知此体系中，以平均速率 $\bar{v}$ 代替瞬时速率 $v_{0}$ 的相对误差绝对值一定 $\leq 0.0443 \%$ 。

按照上面的方法, 计算其他浓度配比的反应体系中以平均速率 $\bar{v}$ 代替瞬时速率 $v_{0}$ 的相对误差绝 对值，见表 1 。

由表 1 中可知, 3 号加入丙酮的体积最小, $\bar{v}$ 与 $v_{0}$ 相对误差的绝对值最大, 但也只有 $0.0885 \%$, 说明这个实验中用 $0-t$ 时间段平均速率代替 0 时刻瞬时速率带来的方法误差比较小。相比于用量筒量 取体积产生的误差也是很小的, 讨论误差来源时不考虑其他因素, 只提出这个是主要原因是不对的。 
表 1 丙酮碘化反应不同体系中 $\bar{v}$ 与 $v_{0}$ 的相对误差

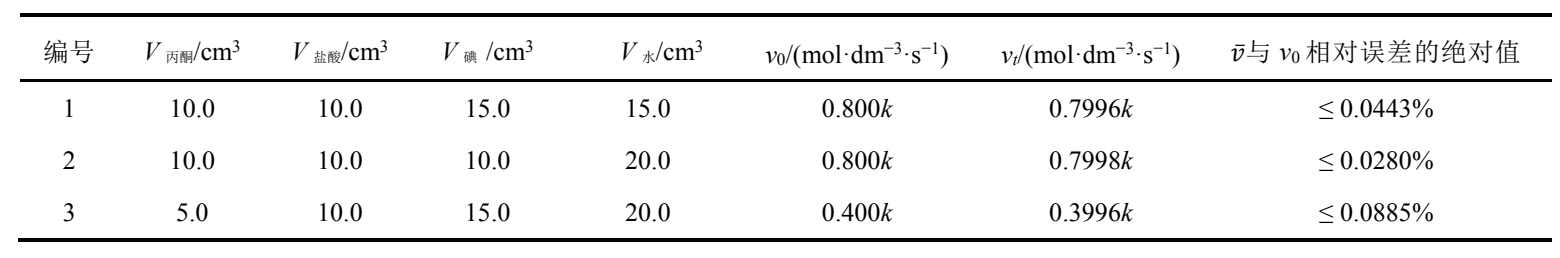

下面我们来讨论一下为什么这个实验中用平均速率代替瞬时速率带来的误差如此之小。

此体系中, 丙酮初始量为 $4.00 \times 10.0 \times 10^{-3}=4.00 \times 10^{-2}(\mathrm{~mol})$, 根据反应方程 1.1 的计量关系, $t$ 时刻碘全部反应完成消耗的丙酮量为: $0.00118 \times 15.0 \times 10^{-3}=1.77 \times 10^{-5}(\mathrm{~mol})$, 占丙酮初始量的比 例为 $\frac{1.77 \times 10^{-5}}{4.00 \times 10^{-2}} \times 100 \%=0.0443 \%$ 。在图 1 上是离开初始浓度很小的一段, 可以看作一段斜线而不是 指数衰减线, 在斜线上任何时刻瞬时速率都相等, 也等于平均速率。

对丙酮碘化反应, 用瞬时速率代替平均速率, 但误差却很小的根本原因是实验设计中, 碘的浓 度相比于丙酮小很多, 碘全部反应完的时间内消耗的丙酮相比较其原始浓度可以忽略不计; 从另一 个视角来看, 丙酮反应浓度变化很小也可以理解为对丙酮来讲反应时间非常短, 与理论课上讨论瞬 时速率与平均速率的关系时说 “当反应时间无限小的时候平均速率等于瞬时速率” 相符合。

对于另一个常见的过二硫酸铵与碘化钾反应体系, 也可以通过关注在计时时间开始和结束时反 应物的浓度，估算两种速率之间误差大小关系。

综上所述, 因为在测量时间段内丙酮浓度改变量非常少, 所以丙酮碘化反应实验中用平均速率 代替瞬时速率带来的实验误差是非常小的, 仅为 $10^{-4}$ 数量级, 充分说明科学不能想当然, 应该经过 深入研究才能下结论。

\section{参 考 文 献}

[1] 陈虹锦, 马荔, 黄梦娇. 实验化学(上册). 第 2 版. 北京: 科学出版社, 2007: 233-235.

[2] 范勇, 曲学俭, 徐家宁. 基础化学实验. 第 2 版. 北京: 高等教育出版社, 2015: 84-85.

[3] 郭伟强, 主编. 大学化学基础实验. 第 2 版. 北京: 科学出版社, 2010: 143-145.

[4] 沈建中, 马林, 赵滨, 卫景德. 普通化学实验. 上海: 复旦大学出版社, 2007: 108-109.

[5] 北京师范大学无机化学教研室等. 无机化学实验. 第 3 版. 北京: 高等教育出版社, 2006: 117-118.

[6] 陈六平, 邹世春, 主编. 现代化学实验与技术. 北京: 科学出版社, 2010: 241-243.

[7] 李梅, 韩莉, 梁竹梅. 化学实验与生活. 第 2 版. 北京: 化学工业出版社, 2017: 122-124. 